\title{
Robust Backstepping Control of Variable Speed Wind Turbines with Permanent Magnet Synchronous Generators
}

\author{
Murat Seker, Erkan Zergeroglu, and Enver Tatlicioglu
}

\begin{abstract}
In this study, we present a robust backstepping approach for the control problem of the variable speed wind turbine with permanent magnet synchronous generator. Specifically to overcome the negative effects of parametric uncertainty, of both mechanical and electrical sub-systems, a differentiable robust controller has been proposed. The proposed methodology ensures the generator velocity tracking error to uniformly approach a small bound where practical tracking is achieved. Stability of the overall system is ensured by Lyapunov-based analysis techniques. Simulation studies are presented to illustrate the feasibility and efficiency of the proposed controller.
\end{abstract}

\section{INTRODUCTION}

When compared to the other sources of energy used for electricity generation, wind is free and plentiful in supply, therefore is attractive in terms of cost and energy security. Owing to these and its reduced environmental problems, electrical energy generation from wind power is a growing sector in the electricity production industry. Among other types of wind turbines, variable speed wind turbines maximize the energy capture by operating the turbine at the peak of the power coefficient. The main aim of variable speed wind turbines is to follow wind velocity variations in low and moderate speeds to maximize aerodynamic efficiency, so have the potential to maximize energy generation. However the quality of power generation and output regulation strongly depends on the control technique employed [1]. That is the effectiveness and reliability of the wind power generation changes depending on the control strategy, and to make wind power truly cost-effective and reliable for variable speed wind turbines, use of advanced control techniques are imperative. To increase the efficiency, model based control design approaches can be applied. One drawback, however, is that mechanical and electrical parameter values of wind turbines are usually not truly available. Especially in practical applications, uncertainties limit the efficient energy capture of a wind turbine. In the literature, different control strategies have been proposed for variable speed wind turbines [1], [2], [3], [4], [5], [6], [7]. In [1], Muldaji et al. evaluated a variable speed, stall-regulated strategy which eliminates the need for ancillary aerodynamic control systems. In [2], a cascade structure nonlinear controller has been proposed, however the proposed mechanism did not account for parametric

M. Seker and E. Zergeroglu are with the Department of Computer Engineering, Gebze Inst. of Tech., 41400, Gebze, Kocaeli, Turkey.

[mseker, ezerger] @bilmuh.gyte.edu.tr

E. Tatlicioglu is with the Department of Electrical \& Electronics Engineering, Izmir Inst. of Tech., 35430, Izmir, Turkey.

envertatliciogluaiyte.edu.tr uncertainties of the system. In [4], Song et al. presented two nonlinear controllers, one of them being an exact model knowledge controller and the other one is an adaptive controller for the rotor velocity tracking. However the proposed adaptive controller scheme could only compensate for the uncertainties in the mechanical sub-system and required the exact knowledge of electrical sub-system parameters. In [5] and [6], the results in [4] was extended to compensate for the uncertainties of both electrical and mechanical sub-systems. In [6], the issues of external disturbances and modelling errors were also addressed.

In this paper, we have extended our previous results given in [5] and [6] to a more sophisticated variable speed wind turbine model, and present a novel robust backstepping approach for the control problem of the variable speed wind turbine with permanent magnet synchronous generator. Specifically to overcome the negative effects of parametric uncertainty, of both mechanical and electrical sub-systems, a differentiable robust controller has been proposed. The proposed methodology ensures the generator velocity tracking error to uniformly approach a small bound where practical tracking is achieved. Simulation studies are presented to illustrate the feasibility and efficiency of the proposed controller.

The rest of the paper is organized as follows. In Section II, the model of the wind turbine used in this study, and the problem statement are given. The error system development and the robust backstepping controller design scheme are presented in Section III. The stability and boundedness of the closed-loop system are investigated in Section IV. While the simulation studies and concluding remarks are given in Sections V and VI, respectively.

\section{DYNAMICAL MODEL}

The mathematical equations describing the dynamics of a variable speed wind turbine with permanent magnet sychronous generator in $d-q$ coordinates is assumed to have the following form [8]

$$
\begin{aligned}
L_{d} \frac{d i_{d}}{d t} & =-R_{s} i_{d}-L_{q} i_{q} \omega_{g}+k_{g} \lambda_{m} \omega_{g}-v_{d} \\
L_{q} \frac{d i_{q}}{d t} & =L_{d} i_{d} \omega_{g}-R_{s} i_{q}-v_{q} \\
J \frac{d \omega_{g}}{d t} & =-\frac{1.5 P^{2}}{4} \lambda_{m} i_{d}-\frac{B P}{2} \omega_{g}+\frac{P}{2} T_{m}
\end{aligned}
$$

where the first equation is for the mechanical sub-system, and the last two equations represent electrical sub-system dynamics. Specifically, $v_{d}(t), v_{q}(t) \in \mathbb{R}$, and $i_{d}(t), i_{q}(t) \in$ 
$\mathbb{R}$ are the general $d-q$ terminal voltages and currents, respectively, $R_{s} \in \mathbb{R}$ is the stator resistance, $L_{d}, L_{q} \in \mathbb{R}$ are the $d-$ and $q-$ axes inductances, respectively, $\lambda_{m} \in \mathbb{R}$ is the permanent magnet flux, $k_{g} \in \mathbb{R}$ is the generator coefficient, $T_{m}(t) \in \mathbb{R}$ is the input mechanical torque of the wind turbine, $\omega_{g}(t) \in \mathbb{R}$ is the generator velocity, $P \in \mathbb{R}$ is the number of poles, $J \in \mathbb{R}$ is the rotor moment of inertia, $B \in \mathbb{R}$ is the friction and windage coefficient.

Our control objective is to ensure that the generator velocity, $\omega_{g}(t)$, tracks a smooth reference velocity profile, denoted by $\omega_{d}(t) \in \mathbb{R}$, generated according to the operational modes of the wind turbine, despite the lack of exact knowledge of both the mechanical and electrical sub-system parameters of (1), (2) and (3).

\section{ERRor SyStem DEVELOPMENT}

To quantify the control objective, we define error signals, denoted by $e(t), z_{1}(t), z_{2}(t) \in \mathbb{R}$, as follows

$$
\begin{aligned}
e & \triangleq \omega_{d}-\omega_{g} \\
z_{1} & \triangleq i_{d, r e f}-i_{d} \\
z_{2} & \triangleq i_{q, \text { ref }}-i_{q}
\end{aligned}
$$

where $i_{d, \text { ref }}(t), i_{q, \text { ref }}(t) \in \mathbb{R}$ are reference signals that will be designed subsequently. After taking the time derivative of the tracking error term in (4), premultiplying with $J$, and then utilizing (3), we obtain

$$
J \dot{e}=J \dot{\omega}_{d}+\frac{B P}{2} \omega_{g}+\frac{1.5 P^{2}}{4} \lambda_{m} i_{d}-\frac{P}{2} T_{m} .
$$

The above expression can be rewritten in the following simpler form

$$
J \dot{e}=Y \phi+f+\phi_{m} i_{d}
$$

where $f(t) \triangleq-\frac{P}{2} T_{m} \in \mathbb{R}$ contains smooth time-varying uncertainties, $\phi_{m} \triangleq \frac{1.5 P^{2}}{4} \lambda_{m} \in \mathbb{R}$ is an uncertain constant parameter, $Y(t) \triangleq\left[\begin{array}{ll}\dot{\omega}_{d} & \omega_{g}\end{array}\right] \in \mathbb{R}^{1 \times 2}$ contains timevarying functions and $\phi \triangleq\left[\begin{array}{cc}J & \frac{B P}{2}\end{array}\right]^{T} \in \mathbb{R}^{2}$ contains uncertain constant parameters. Based on the definition of $Y(\cdot)$, its desired form is defined as $Y_{d}(t) \triangleq\left[\begin{array}{cc}\dot{\omega}_{d} & \omega_{d}\end{array}\right] \in$ $\mathbb{R}^{1 \times 2}$. After adding and substracting $Y_{d} \phi$ to the right-handside of the open-loop mechanical sub-system dynamics, the following expression can be obtained

$$
J \dot{e}=F+\chi+\widetilde{\phi}_{m} i_{d}+\hat{\phi}_{m} i_{d, r e f}-\hat{\phi}_{m} z_{1}
$$

where $\hat{\phi}_{m} \in \mathbb{R}$ is the best-guess estimate (nominal value) of $\phi_{m}, \widetilde{\phi}_{m} \triangleq \phi_{m}-\hat{\phi}_{m} \in \mathbb{R}$ is the estimation error, and $F(t)$, $\chi(t) \in \mathbb{R}$ are defined as

$$
\begin{aligned}
F & \triangleq Y_{d} \phi+f \\
\chi & \triangleq\left(Y-Y_{d}\right) \phi .
\end{aligned}
$$

Based on the subsequent stability analysis, reference signals $i_{d, r e f}(t)$ and $i_{q, r e f}(t)$ are designed as

$$
\begin{aligned}
i_{d, r e f} & =\frac{-1}{\hat{\phi}_{m}}\left\{Y_{d} \hat{\phi}+\hat{f}+\left(k_{e}+k_{n} \rho_{1}^{2}\right) e+v_{R 1}\right\} \\
i_{q, \text { ref }} & =0
\end{aligned}
$$

where $k_{e}, k_{n} \in \mathbb{R}$ are positive control gains, $\hat{\phi}, \hat{\phi}_{m}, \hat{f} \in$ $\mathbb{R}$ are the constant best-guess estimates of the unknown parameters $\phi, \phi_{m}$, and $f$, respectively, the positive bounding function $\rho_{1}(e) \in \mathbb{R}$ is designed to satisfy

$$
\rho_{1} \geq|\chi|
$$

and $v_{R 1}(t) \in \mathbb{R}$ is a robust term designed in the following form [9]

$$
v_{R 1}=\frac{e \rho_{2, s}^{2}}{\|e\|_{m} \rho_{2, m}+\epsilon_{1}}
$$

where $\epsilon_{1} \in \mathbb{R}$ is a positive constant, and $\rho_{2}(|e|) \in \mathbb{R}$ is a positive bounding function that is designed to satisfy

$$
\rho_{2}(|e|) \geq|\widetilde{F}|
$$

where $\widetilde{F}(t) \triangleq F-Y_{d} \hat{\phi}-\hat{f} \in \mathbb{R}$. In (15), $\rho_{2, s} \triangleq \rho_{2}\left(\|e\|_{s}\right) \in$ $\mathbb{R}$ and $\rho_{2, m} \triangleq \rho_{2}\left(\|e\|_{m}\right) \in \mathbb{R}$ are auxiliary positive bounding functions where the notations $\|e\|_{s}$ and $\|e\|_{m}$ are used to define the following functions

$$
\|e\|_{s} \triangleq \sqrt{e^{2}+\sigma},\|e\|_{m} \triangleq \sqrt{e^{2}+\sigma}-\sqrt{\sigma}
$$

where $\sigma \in \mathbb{R}$ is a small, positive constant. Note that, based on the definitions in (17), the following inequalities are always satisfied

$$
\begin{aligned}
& \|e\|_{s} \geq \quad|e| \geq\|e\|_{m} \\
& \rho_{2}\left(\|e\|_{s}\right) \geq \rho_{2}(|e|) \geq \rho_{2}\left(\|e\|_{m}\right) \text {. }
\end{aligned}
$$

Remark 1: The backstepping procedure requires that the auxiliary control in (12) be differentiable; hence, the robust control term in (15) was defined with the functions given by (17) to ensure differentiability.

After inserting $i_{d, r e f}(t)$ back into the open-loop mechanical sub-system dynamics, we obtain

$$
J \dot{e}=-k_{e} e-k_{n} \rho_{1}^{2} e+\chi-\hat{\phi}_{m} z_{1}+\widetilde{F}+\widetilde{\phi}_{m} i_{d}-v_{R 1} .
$$

Note that the term $\widetilde{\phi}_{m} i_{d}$ can be upper bounded as

$$
\rho_{3}\left|z_{1}\right| \geq\left|\widetilde{\phi}_{m} i_{d}\right|
$$

where $\rho_{3}(t) \in \mathbb{R}$ is a positive bounding function. The backstepping design also requires the dynamics of the auxiliary error signals $z_{1}(t)$ and $z_{2}(t)$. To obtain the dynamics for $z_{1}(t)$, first the derivative of (5) is taken, and then the time derivative of (12) is inserted and the resulting expression is multiplied with $L_{d}$ to obtain

$$
\begin{aligned}
L_{d} \dot{z}_{1}= & \frac{-L_{d}}{\hat{\phi}_{m}}\left\{\dot{Y}_{d} \hat{\phi}+\frac{1}{J}\left(k_{e}+2 k_{n} \rho_{1} e \frac{\partial}{\partial e} \rho_{1}+k_{n} \rho_{1}^{2}\right.\right. \\
& \left.\left.+\frac{\partial}{\partial e} v_{R 1}\right) \times\left(Y \phi+f+\phi_{m} i_{d}\right)\right\} \\
& +R_{s} i_{d}+L_{q} i_{q} \omega_{g}+v_{d}-k_{g} \lambda_{m} \omega_{g}
\end{aligned}
$$

which can be rewritten in the following manner

$$
L_{d} \dot{z}_{1}=W_{1} \theta_{1}+f_{1}+v_{d}
$$


where $W_{1}(\cdot) \theta_{1} \in \mathbb{R}$ contains the linearly parametrizable terms with $W(t) \in \mathbb{R}^{1 \times 6}$ and $\theta_{1} \in \mathbb{R}^{6}$ containing timevarying functions and uncertain constant parameters, respectively, and $f_{1}(t) \in \mathbb{R}$ contains the time-varying uncertainties, and are explicitly defined as follows

$$
\begin{aligned}
W_{1} \theta_{1}= & \frac{-L_{d}}{\hat{\phi}_{m}}\left\{\dot{Y}_{d} \hat{\phi}+\frac{1}{J}\left(k_{e}+2 k_{n} \rho_{1} e \frac{\partial}{\partial e} \rho_{1}+k_{n} \rho_{1}^{2}\right.\right. \\
& \left.\left.+\frac{\partial}{\partial e} v_{R 1}\right) \times\left(Y \phi+\phi_{m} i_{d}\right)\right\} \\
& +R_{s} i_{d}+L_{q} i_{q} \omega_{g}-k_{g} \lambda_{m} \omega_{g} \\
f_{1}=\frac{-L_{d}}{\hat{\phi}_{m}} & \frac{1}{J}\left(k_{e}+2 k_{n} \rho_{1} e \frac{\partial}{\partial e} \rho_{1}+k_{n} \rho_{1}^{2}+\frac{\partial}{\partial e} v_{R 1}\right) f .
\end{aligned}
$$

Based on the subsequent stability analysis, we design $v_{d}(t)$ in the form

$$
v_{d}=-\left(k_{1}+k_{n} \rho_{3}^{2}\right) z_{1}-W_{1} \hat{\theta}_{1}+\hat{\phi}_{m} e-v_{R 2}
$$

where $\hat{\theta}_{1} \in \mathbb{R}^{6 \times 1}$ is the constant best-guess estimate of the unknown parameter vector $\theta_{1}, v_{R 2}(t) \in \mathbb{R}$ is an additional robust control term, $k_{1} \in \mathbb{R}$ is a positive constant control gain, and $\rho_{3}(\cdot)$ was introduced in (21). In (26), the robust term $v_{R 2}(t)$ has been introduced to compensate for the mismatch between the actual and estimated parameters and is explicitly designed as follows

$$
v_{R 2}=\frac{z_{1} \rho_{4}^{2}}{\left|z_{1}\right| \rho_{4}+\epsilon_{2}}
$$

where $\epsilon_{2} \in \mathbb{R}$ is a positive constant, and $\rho_{4}(t) \in \mathbb{R}$ is a positive bounding function designed to satisfy

$$
\rho_{4} \geq\left|W_{1} \widetilde{\theta}_{1}\right|+\left|f_{1}\right|
$$

where $\widetilde{\theta}_{1} \triangleq \theta_{1}-\hat{\theta}_{1} \in \mathbb{R}^{6 \times 1}$ is the parameter estimation error. After substituting (26) into (22), the closed-loop dynamics for $z_{1}(t)$ is obtained to have the following form

$$
L_{d} \dot{z}_{1}=-k_{1} z_{1}-k_{n} \rho_{3}^{2} z_{1}+W_{1} \widetilde{\theta}_{1}+f_{1}+\hat{\phi}_{m} e-v_{R 2} .
$$

Similarly, the dynamics of $z_{2}(t)$ can be obtained to have the following form

$$
L_{q} \dot{z}_{2}=-L_{d} i_{d} \omega_{g}+R_{s} i_{q}+v_{q}
$$

After defining $W_{2}(t) \triangleq\left[\begin{array}{ll}-i_{d} \omega_{g} & i_{q}\end{array}\right] \in \mathbb{R}^{1 \times 2}$ which contains time-varying functions, and $\theta_{2} \triangleq\left[\begin{array}{ll}L_{d} & R_{s}\end{array}\right]^{T} \in$ $\mathbb{R}^{2}$ which contains uncertain constant parameters, the righthand-side of (30) can be rewritten as

$$
L_{q} \dot{z}_{2}=W_{2} \theta_{2}+v_{q}
$$

Similar to the design of $v_{d}(t)$, the voltage input $v_{q}(t)$ is designed in the following form

$$
v_{q}=-k_{2} z_{2}-W_{2} \hat{\theta}_{2}-v_{R 3}
$$

where $k_{2} \in \mathbb{R}$ is a positive constant control gain, $\hat{\theta}_{2} \in \mathbb{R}^{2 \times 1}$ is the constant best-guess estimate of the unknown parameter vector $\theta_{2}$, and $v_{R 3}(t) \in \mathbb{R}$ is an additional robust control term introduced to compensate for the mismatch between the actual and estimated parameters and is explicitly defined as follows

$$
v_{R 3}=\frac{z_{2} \rho_{5}^{2}}{\left|z_{2}\right| \rho_{5}+\epsilon_{3}}
$$

where $\epsilon_{3} \in \mathbb{R}$ is a positive constant, and $\rho_{5}(t) \in \mathbb{R}$ is a positive bounding function that is designed to satisfy

$$
\rho_{5} \geq\left|W_{2} \widetilde{\theta}_{2}\right|
$$

where $\widetilde{\theta}_{2} \triangleq \theta_{2}-\hat{\theta}_{2} \in \mathbb{R}^{2}$ is the parameter estimation error. Substituting (32) into (30), the closed-loop dynamics for $z_{2}(t)$ is obtained to have the following form

$$
L_{q} \dot{z}_{2}=-k_{2} z_{2}+W_{2} \widetilde{\theta}_{2}-v_{R 3}
$$

\section{STABILITy ANALYSis}

Forming the closed-loop error dynamics for the signals $e(t), z_{1}(t)$, and $z_{2}(t)$, we are now ready to state the following theorem.

Theorem 1: The robust controller given by (26), (32) and the auxiliary control inputs (12), (13) with the robust terms (15), (27), and (33) guarantees uniformly ultimately boundedness of the generator velocity tracking error signal $e(t)$ in the sense that

$$
\|e(t)\| \leq \sqrt{\frac{a}{b}\|x(0)\|^{2} \exp (-\beta t)+\frac{2 \epsilon}{b \beta}(1-\exp (-\beta t))}
$$

where $x \triangleq\left[\begin{array}{ccc}e & z_{1} & z_{2}\end{array}\right]^{T} \in \mathbb{R}^{3 \times 1}$ is the combined error signal, and $a, b, \beta, \epsilon \in \mathbb{R}$ are positive constants defined as

$$
\begin{aligned}
& a \triangleq \max \left\{J, L_{d}, L_{q}\right\} \\
& b \triangleq \min \left\{J, L_{d}, L_{q}\right\} \\
& \beta \triangleq \frac{2 \min \left\{\left(k_{e}-\frac{1}{4 k_{n}}\right), k_{1}, k_{2}\right\}}{\max \left\{J, L_{d}, L_{q}\right\}} \\
& \epsilon \triangleq \epsilon_{1}+\epsilon_{2}+\epsilon_{3}+\frac{1}{4 k_{n}}
\end{aligned}
$$

where $\epsilon_{1}, \epsilon_{2}, \epsilon_{3}, k_{e}, k_{1}, k_{2}$, and $k_{n}$ were previously defined.

Proof: We start our proof by defining the following non-negative scalar function

$$
V \triangleq \frac{1}{2} J e^{2}+\frac{1}{2} L_{d} z_{1}^{2}+\frac{1}{2} L_{q} z_{2}^{2}
$$

which can be lower and upper bounded in the following from

$$
\frac{1}{2} \min \left\{J, L_{d}, L_{q}\right\}\|x\|^{2} \leq V \leq \frac{1}{2} \max \left\{J, L_{d}, L_{q}\right\}\|x\|^{2}
$$


Taking the time derivative of (41) along (20), (29) and (35), and then cancelling common terms, we obtain

$$
\begin{aligned}
\dot{V}= & -k_{e} e^{2}-k_{1} z_{1}^{2}-k_{2} z_{2}^{2} \\
& +\left[\chi-k_{n} \rho_{1}^{2} e\right] e \\
& +\left[\widetilde{F}-v_{R 1}\right] e \\
& +\left[\widetilde{\phi}_{m} i_{d} e-k_{n} \rho_{3}^{2} z_{1}^{2}\right] \\
& +\left[W_{1} \widetilde{\theta}_{1}+f_{1}-\frac{z_{1} \rho_{4}^{2}}{\left|z_{1}\right| \rho_{4}+\epsilon_{2}}\right] z_{1} \\
& +\left[W_{2} \widetilde{\theta}_{2}-\frac{z_{2} \rho_{5}^{2}}{\left|z_{2}\right| \rho_{5}+\epsilon_{3}}\right] z_{2} .
\end{aligned}
$$

It should be noted that, the $\left[\widetilde{F}-v_{R 1}\right] e$ term can be upper bounded as [9]

$$
\begin{aligned}
{\left[\widetilde{F}-v_{R 1}\right] e } & \leq \rho_{2}|e|-\frac{e^{2} \rho_{2, s}^{2}}{\|e\|_{m} \rho_{2, m}+\epsilon_{1}} \\
& \leq \rho_{2}|e|-\frac{e^{2} \rho_{2}^{2}}{|e| \rho_{2}+\epsilon_{1}} \\
& \leq \epsilon_{1} \frac{|e| \rho_{2}}{|e| \rho_{2}+\epsilon_{1}} \leq \epsilon_{1} .
\end{aligned}
$$

After using (14), (16), (21), (28), and (34), we can upper bound the right-hand-side of (43) as follows

$$
\begin{aligned}
\dot{V} \leq & -k_{e} e^{2}-k_{1} z_{1}^{2}-k_{2} z_{2}^{2} \\
& +\left[\rho_{1}|e|-k_{n} \rho_{1}^{2} e^{2}\right] \\
& +\left[\rho_{3}\left|z_{1}\right||e|-k_{n} \rho_{3}^{2} z_{1}^{2}\right] \\
& +\left[\rho_{4}\left|z_{1}\right|-\frac{z_{1}^{2} \rho_{4}^{2}}{\left|z_{1}\right| \rho_{4}+\epsilon_{2}}\right] \\
& +\left[\rho_{5}\left|z_{2}\right|-\frac{z_{2}^{2} \rho_{5}^{2}}{\left|z_{2}\right| \rho_{5}+\epsilon_{3}}\right]+\epsilon_{1} .
\end{aligned}
$$

After adding and subtracting $\frac{1}{4 k_{n}}$ and then completing the squares of the first and second bracketed terms of (45), we can further upper bound (45) as

$$
\begin{aligned}
\dot{V} \leq & -\min \left\{\left(k_{e}-\frac{1}{4 k_{n}}\right), k_{1}, k_{2}\right\}\|x\|^{2} \\
& +\left[\rho_{4}\left|z_{1}\right|-\frac{z_{1}^{2} \rho_{4}^{2}}{\left|z_{1}\right| \rho_{4}+\epsilon_{2}}\right] \\
& +\left[\rho_{5}\left|z_{2}\right|-\frac{z_{2}^{2} \rho_{5}^{2}}{\left|z_{2}\right| \rho_{5}+\epsilon_{3}}\right]+\frac{1}{4 k_{n}}+\epsilon_{1}
\end{aligned}
$$

where we used

$$
\rho_{3}\left|z_{1}\right||e|-k_{n} \rho_{3}^{2} z_{1}^{2} \leq \frac{e^{2}}{4 k_{n}} .
$$

The bracketed terms in (46) can be manipulated as follows

$$
\rho_{i+3}\left|z_{i}\right|-\frac{\rho_{i+3}^{2} z_{i}^{2}}{\rho_{i+3}\left|z_{i}\right|+\epsilon_{i+1}}=\epsilon_{i+1} \frac{\rho_{i+3}\left|z_{i}\right|}{\rho_{i+3}\left|z_{i}\right|+\epsilon_{i+1}} \leq \epsilon_{i+1}
$$

eqnarray where $i \in\{1,2\}$. Hence, we can use (48) to place an upper bound on the right-hand-side of (46) as shown below

$$
\dot{V} \leq-\min \left\{\left(k_{e}-\frac{1}{4 k_{n}}\right), k_{1}, k_{2}\right\}\|x\|^{2}+\epsilon
$$

where $\epsilon$ was previously defined in (40). From the upper bound on $V(t)$ given in (42), we can further upper bound $\dot{V}(t)$ in (49) as shown below

$$
\dot{V} \leq-\beta V+\epsilon
$$

where $\beta$ was previously defined in (39). The differential inequality of (50) can now be solved to yield [9]

$$
V(t) \leq V(0) \exp (-\beta t)+\frac{\epsilon}{\beta}(1-\exp (-\beta t)) .
$$

After applying the bounds of (42) to (51), we obtain the following upper bound for $x(t)$

$$
\|x(t)\| \leq \sqrt{\frac{a}{b}\|x(0)\|^{2} \exp (-\beta t)+\frac{2 \epsilon}{b \beta}(1-\exp (-\beta t))}
$$

where $a, b$ were previously defined in (37), (38), respectively. Based on (52) and the definition of $x(t)$, we can show that the generator velocity tracking error $e(t)$ can be bounded as given by (36) [10]. Due to the boundedness of $e(t), z_{1}(t)$, and $z_{2}(t)$, standard signal chasing arguments can be utilized to show that all the signals in the closed-loop systems in (20), (29) and (35) are bounded.

\section{Simulation Results}

To demonstrate the performance of the proposed robust controller, two different simulation studies were performed similar to that of [4]. For the first simulation, the reference generator velocity $\omega_{d}(t)$ was selected as

$$
\omega_{d}(t)=2+\sin (t)
$$

and for the second one, a more realistic reference generator velocity was selected as

$$
\omega_{d}(t)= \begin{cases}0, & u(k)<u_{c}, \\ x_{m}\left(1+\sin \left(\frac{\pi}{2} \frac{\left(u(k)-s_{1}\right)}{d_{1}}\right)\right), & u(k)<u_{r}, \\ x_{m}, & u(k)<u_{F}, \\ x_{m}\left(1+\sin \left(\frac{\pi}{2} \frac{\left(u(k)-s_{2}\right)}{d_{2}}\right)\right), & u(k)<u_{s}, \\ 0, & u(k)>u_{s}\end{cases}
$$

with

$$
\begin{gathered}
s_{1}=\frac{u_{c}+u_{r}}{2}, \quad d_{1}=\frac{u_{r}-u_{c}}{2}, \\
s_{2}=\frac{u_{F}+u_{r}}{2}, d_{2}=\frac{u_{r}-u_{F}}{2}, \\
u_{s}=21.3 \mathrm{~m} / \mathrm{sec}, x_{m}=4.1 \mathrm{rad} / \mathrm{sec}, \\
u_{c}=4.3 \mathrm{~m} / \mathrm{sec} u_{r}=7.7 \mathrm{~m} / \mathrm{sec} u_{F}=17.9 \mathrm{~m} / \mathrm{sec} .
\end{gathered}
$$

where that the parameter $x_{m}$ is specified according to the allowable generator velocity. The system parameters used in the simulations were

$$
\begin{aligned}
L_{d} & =0.002, L_{q}=0.002, R_{s}=0.18, k_{g}=100, \\
J & =0.48, B=0.001, \lambda=0.8, p=8
\end{aligned}
$$

For both simulations, the best-guess estimates of the parameters were set to $80 \%$ of the actual values and controller gains were chosen as

$$
\begin{aligned}
& k_{e}=0.01, k_{n}=1000, k_{1}=0.001, k_{2}=35 \\
& \rho_{1}=1.4, \rho_{2}=7.1, \rho_{3}=13.1, \rho_{4}=30, \rho_{5}=10,(57) \\
& \varepsilon_{1}=0.001, \varepsilon_{2}=0.001, \varepsilon_{3}=0.00001 .
\end{aligned}
$$


The results of the simulations are presented through Figures 1-4. For the first simulation, the desired and actual shaft velocity profiles and the tracking errors are given in Figure 1 , and the control inputs $v_{d}(t)$ and $v_{q}(t)$ are given in Figure 2. Similarly, the results of second simulation, where a more realistic desired velocity profile was preferred, are given in Figures 3 and 4, respectively. From Figures 1 and 3, it is clear that tracking objective was met.

\section{CONCLUSions}

In this paper, we have presented a robust backstepping controller scheme for the variable speed wind turbines with permanent magnet synchronous generators. The proposed method achieved practical tracking of the generator velocity error despite the parametric uncertainty on both mechanical and electrical sub-systems. That is, the generator velocity tracking error was driven, uniformly, to approach to a small bound. The boundedness of the system states and stability of the closed-loop system were guaranteed via Lyapunov-based techniques. Simulation studies were presented to illustrate the performance and feasibility of the proposed method.
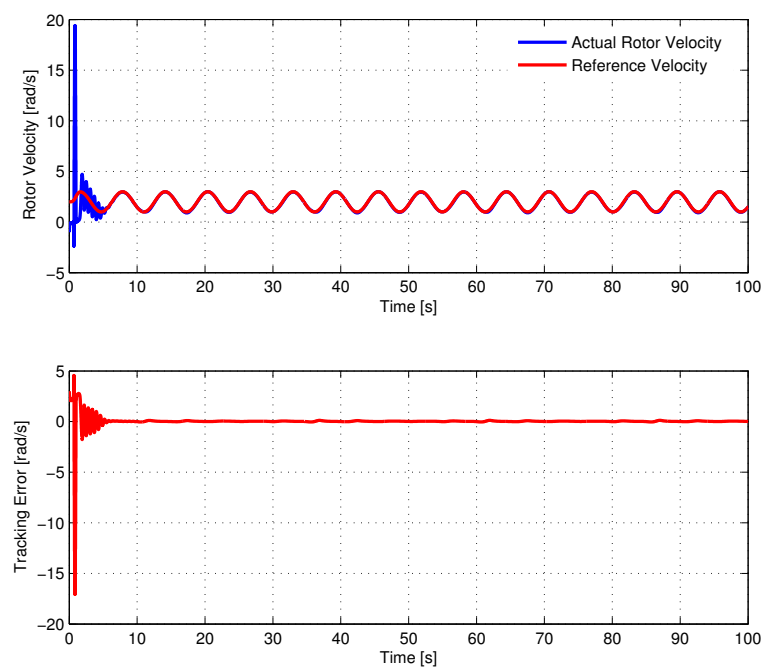

Fig. 1. (First Simulation) (Top) Actual and reference generator velocity profiles, (Bottom) Tracking error $e(t)$

\section{REFERENCES}

[1] E. Muldaji, K. Pierce, and P. Migliore, "Control strategy for variablespeed, stall-regulated wind turbines, stall-regulated wind turbines," in Proc. American Control Conf., Philadelphia, PA, USA, 1998, pp. $1710-1714$.

[2] B. Boukhezzar and H. Siguerdidjane, "Nonlinear control of wind turbines for power regulation," in Proc. of IEEE Int. Conf. on Control Applications, Toronto, Canada, 2005, pp. 114-119.

[3] K. E. Johnson, Y. P. Lucy, M. J. Balas, and L. J. Fingersh, "Control of variable speed wind turbines," IEEE Control Syst. Mag., vol. 26, no. 3, pp. 70-81, 2006.

[4] Y. D. Song, B. Dhinarakaran, and X. Y. Bao, "Variable speed control of wind turbines using nonlinear and adaptive algorithms," Journal of Wind Engineering and Industrial Aerodynamics, vol. 85, pp. 293-308, 2000.
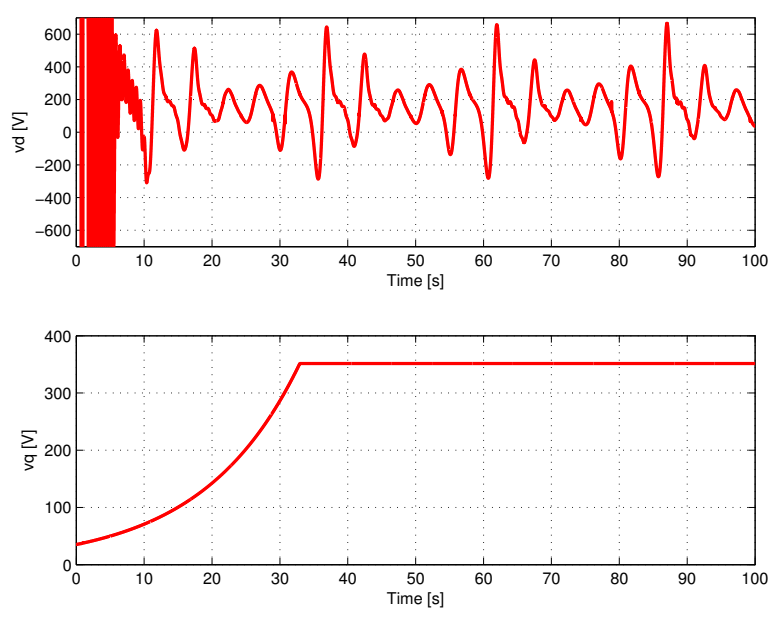

Fig. 2. (First Simulation) Voltages applied to the generator: (Top) $v_{d}(t)$, (Bottom) $v_{q}(t)$
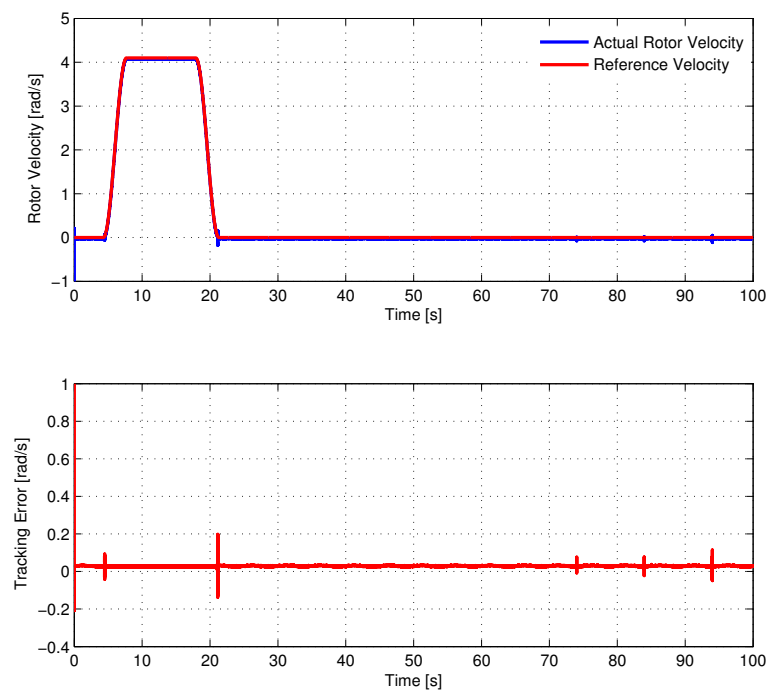

Fig. 3. (Second Simulation) (Top) Actual and reference generator velocity profiles, (Bottom) Tracking error $e(t)$

[5] U. Ozbay, E. Zergeroglu, and S. Sivrioglu, "Adaptive backstepping control of variable speed wind turbines," Int. J. Control, vol. 81, no. 6, pp. 910-919, 2008.

[6] S. Sivrioglu, U. Ozbay, and E. Zergeroglu, "Variable speed control of wind turbines: A robust backstepping approach," in Proc. of the 17th World Congress The International Federation of Automatic Control, B10. Seoul Korea, July 2008, pp. 1183-1188.

[7] E. Iyasere, D. M. Dawson, J. R. Wagner, M. H. Salah, and E. Tatlicioglu, "Nonlinear robust control to maximize energy capture in a variable speed wind turbine using an induction generator," in Proc. of Int. Conf. on Systems, Man and Cybernetics, San Antonio, TX, USA, 2009, pp. 4171-4176.

[8] A. Abedini and H. Nikkhajoei, "Dynamic model and control of a wind-turbine generator with energy storage," IET Renewable Power Generation, vol. 5, no. 1, pp. 67-78, 2011.

[9] D. M. Dawson, M. M. Bridges, and Z. Qu, Nonlinear Control of Robotic Systems for Environmental Waste and Restoration. Englewood Cliffs, NJ, USA: Prentice Hall, 1995.

[10] D. M. Dawson, J. Hu, and T. C. Burg, Nonlinear Control of Electric 

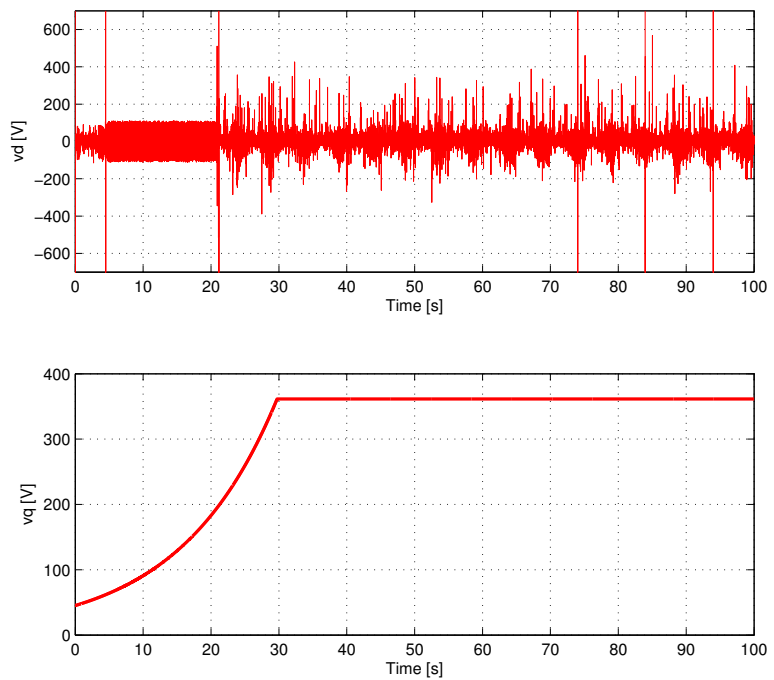

Fig. 4. (First Simulation) Voltages applied to the generator: (Top) $v_{d}(t)$, (Bottom) $v_{q}(t)$

Machinery. New York, NY, USA: Marcel Dekker, 1998. 